\title{
DIVERCITY: Distributed Virtual Workspace For Enhancing Communication and Collaboration Within the Construction Industry
}

\author{
Y. Arayici \& G. Aouad \\ School of Construction and Property Management, University of Salford, Greater Manchester, UK
}

\begin{abstract}
DIVERCITY is a large EU funded project in the area of construction IT undertaken by a European consortium of researchers and practitioners from the construction industry. It is the acronym for Distributed Virtual Workspace for enhancing Communication within the Construction Industry and the prototype that presents the mechanism to smoothly and collaboratively conduct the construction projects from early briefing to the detailed design and even further by the end of the construction phase. To be precise, DIVERCITY aims to supply a shared virtual construction design and briefing environment that enables the construction industry to better undertake the client briefing and design review phases of a project.
\end{abstract}

DIVERCITY comprises three main workspaces, which are client briefing, design review and construction workspaces respectively. Whilst the Client Briefing workspace enables architect to interact and communicate with client for capturing client needs, the design review workspace allows design team to review the design solution in different respects such as lighting, acoustic and thermal conditions and the construction workspace helps the planner evaluate optimum buildability for a building through communication with other parties of the design team and site planning and management.

The paper presents the DIVERCITY system and its main six components: Client Briefing, Lighting, Acoustic, Thermal and Heating Simulations, Visual Product Chronology for construction planning, lastly Site planning \& Analysis, how each of them handles different aspects of a construction project in a construction supply chain and how they complement each others to constitute a seamless integrated computer environment for the sake of excellence of briefing and design and construction planning.

\section{INTRODUCTION}

The Construction industry is one of the major sectors with 780 billion Euros: it means that the construction industry is the largest industry in the industrial employment in Europe with 11 million workers, which equals $7 \%$ of the working population. Furthermore, owing to being dependent on the construction industry, 22 million jobs are created in other sectors (Coudret et al, 2001).

In the past decade, construction companies have spent a great deal of effort and resources in improving their business processes. New forms of innovative project management, supported by recent IT developments, have appeared in response to evergrowing pressure from owners to complete projects on time and deliver high quality buildings (Sarshar \& Christiansson, 2004).
Construction has become an information intensive industry; and a new activity has emerged from the process of managing projects, establishing itself as a discipline in its own right: information management (Construct IT 2000).

Despite the interest and effort applied by leading companies, information management in the construction industry is still in its infancy (Sarshar \& Christiansson, 2004). Construction projects involve a large number of direct stakeholders (clients, professional teams, contractors) and indirect stakeholders (local authorities, residents, workers). There are significant barriers to communications between the stakeholders. Many researchers have acknowledged the limitations of current approaches to the management of information in projects (Kiviniemi 1999) (Aouad, 1997) (Alshawi, 1996). Most of these limitations are due to (Sarshar \& Christiansson, 2004): 
- Much project information is stored on paper as drawings and written documents. This is frequently unstructured and difficult to use. It is also easy to lose or damage (Construct IT 2000). Thousands of documents are shared during a typical project, leading to significant human errors in managing the versioning of these documents.

- This process leads to incomplete understanding of the planned construction, functional inefficiencies, inaccurate initial work or clashes between components.

- People responsible for collecting and archiving project data may not always understand the specific needs of those who will use it, such as those involved in building maintenance.

- The data is usually not managed while it is created, but instead it is captured and archived at the end of the construction stage. This means that people who have knowledge about the project are often likely to have left for another project by this time - so their input is not captured.

- Lessons learned are not organised well and are buried in details. It is therefore difficult to compile and disseminate useful knowledge and best practice to other projects (Watson and Marir 1994).

In the past, researchers have used IT for providing numerous decision support systems for the professionals involved in the industry (Faraj \& Alshawi, 1999). However, these systems have created islands of automation and are far from achieving an acceptable level of integration across disciplines and across the design and construction processes (Faraj \& Alshawi, 1999), and (Kartam, 1994).

\section{A VISION FOR CONSTRUCTION IT}

Sarshar (2000) developed a vision for construction IT. Sarshar portrayed a scenario where all stakeholders can produce their relevant project information and post it on an electronic "project information board”. Each user has appropriate access rights and can manipulate the necessary information on demand. This vision has been termed construction "integration", by many researchers (Issa, 1999) (Alshawi, 1996).

In this vision for construction IT (Sarshar 2000), the users of this information board need not be tied to their computers and office network for connections and access. Advances in communications technologies allow users to manipulate information in any format, and in any geographical location. This is known as construction "collaboration" (Sarshar \& Christiansson, 2004).

\subsection{Construction Integration}

Currently, construction project information is captured in documents and 2D CAD drawings. The construction parties may share these documents and drawings using an electronic environment. But problems arise as the volume of documents and drawings and their versions increase (Sarshar 2002).

The "project information board" approach is a means of sharing project information, via a shared conceptual product / process model. Information is entered once and is used by all stakeholders, during a project. Some of the benefits of the integrated approach include:

- Much of the project information can be presented in a visual rather than textual format. This eases communications and information sharing (Issa 1999, Thabet 1999, Brandon 1999).

- Many aspects of the building can be simulated to improve client briefing and design reviews (Sawhney 1999) (Shi 1999).

- Such interactive technology can be used to consider life cycle issues such as environmental impact, space planning, facilities management, emergency evacuation, security and constructability during design reviews. This can facilitate concurrent engineering by involving clients, planners, architects, designers, civil engineers, contractors, facility managers and security personnel (Sarshar 2000,2002).

- It is easier to use past project knowledge and information for new developments (Sarshar \& Christiansson, 2004).

\subsection{Construction Collaboration}

Construction Collaboration is an area, which investigates how the supply chain can access and manipulate the "project information board", irrespective of their geographical location. The key elements of this collaborative environment include (Divercity Handbook 2003, Christiansson et.al.2001):

- Advanced administration tools for distributed personal, team, and project information repositories;

- Access to virtual building models and collaborative environments through wireless 
networked technologies and low cost virtual reality environments;

- Appropriate security levels for sharing the information over the inter/intranets;

- Process and workflow management tools to support variations in working practices between different projects;

- New generations of ICT tools that facilitate

collaboration and communication with end-users.

\section{THE DIVERCITY PROJECT}

DIVERCITY was an EU funded project (19992002) (Divercity Handbook 2003), (Christiansson 2002). The project used IFC standards in order to develop a toolkit for shared virtual briefing and design in the construction industry. This toolkit allows construction companies to conduct client briefing, design reviews, simulate what if scenarios, test constructability of buildings, communicate and co-ordinate design activities between teams. Both synchronous and asynchronous interaction are emphasised in this framework.

DIVERCITY allows users to produce designs and simulate them in a virtual environment. The designs are IFC based and can be viewed by all stakeholders within the project team. The project had the following objectives:

- Creation of a client-briefing workspace, which can facilitate interaction and communication of design ideas between client and the architect

- Creation of an interactive design review workspace, which can facilitate multi-disciplinary design reviews involving different stakeholders of a construction project, i.e. planners, architect, designers, civil engineers, electrical engineers, contractors, facility managers, security personnel etc.

- Creation of a virtual construction workspace, which can assess the buildability (the sequence of construction activities, scheduling, material handling etc) of a building

- Specification and development of a software framework for integrating the above three workspaces and sharing them over networks to support collaboration between geographically distributed project team members.

\section{THE DIVERCITY SYSTEM}

DIVERCITY has developed virtual workspaces that improve communication and collaboration. DIVERCITY has focused on three construction processes, i.e. (i) client briefing; (ii) design reviews; and (iii) site operations and constructability as well as communication and integration framework.

\subsection{Client Briefing Application}

The Client Briefing application; Pre CAD represents the interface between the client and design team: It is the mechanism for communication of ideas, the exploration of concepts and the presentation of the design. It is intended to produce a single initial design, agreed upon by all parties, and as this design is iteratively and progressively turned into a formal detailed design, feedback is obtained in order to drive the design process forward

Recent research carried out at the University of Salford (Barrett, Stanley, 1999) suggests that Client Briefing should not be seen as an event but as a process, which works in an iterative manner to refine the design. Figure 1 shows the DIVERCITY Client Briefing process and a Pre CAD VR environment. In order to achieve this process, the design team needs to be able to present their design to the client in a manner that the client can easily understand. This presentation process may generate new inputs into the design from either the client or the design team. These resultant inputs may be either new parameters for the design, or simple modifications that may be made at the time of the presentation.

\subsection{Design Review Applications}

In the design/Construction process, detailed design is an important phase where the inputs are represented by a rather architectural design (usually drawings on a 1:1000 scale) and the outputs are precise definition of all technical domains related to the design, e.g. structural design, heating and thermal, lighting, acoustic, fire safety, etc (Shelbourn et al, 1999). Although state-of-the-art software tools exist for the detailed design stage, throughout the user requirements capture in the DIVERCITY project, it has been observed that these existing tools suffer from two important limitations: (Shelbourn et al, 1999)

- Discontinuities between the different software tools. This makes the reuse of the results of one technical domain as an input for another technical domain practically impossible;

- Lack of 3D real-time inspection features. Consequently, members of the project team 
spend too much time trying to (i) understand the project information (ii) to describe this information to one another.

In order to greatly reduce the above limitations, in the DIVERCITY context an interactive design review workspace that allows the project teams to visualise and interact with the project on a multidisciplinary basis has been created. The main features of the design review workspace are as follows:

- Supporting continuous design between different phases and within the detailed design phase using IFC (Industry Foundation Classes), which means that the calculation results yielded for one technical domain can be reused as an input for another technical domain.

- Model Driven Approach that allows project teams to share the same view about the project through a visual and shared conceptual model. As a result substantial improvements can then be made on the communication level between the project teams.

Design Review applications within the DIVERCITY scope are:

- Lighting Simulation by means of which user can visualise, with a photo realistic rendering, the lighting conditions of each space taking into account both natural and artificial light sources.

- Acoustic Simulations allowing the user to experience what it would be like to live and work in the spaces of the building

- Heating and Thermal simulations in order to assess both energy consumption of a building and thermal comfort conditions in each space;

\subsubsection{Lighting Simulations}

The lighting simulation module of DIVERCITY provides realistic simulation of light transfers. Moreover, it is the first time that a lighting simulation involving radiosity provides interactive solutions to the user. They can change and move objects or lights in the building and see updated simulation interactively.

The lighting application will enable the user to look at different ways of lighting the spaces by clicking and dragging objects into spaces and placing them at different locations within the space. The reflections and contrasts from surfaces of furniture, walls, windows, etc can be viewed, enabling the user to place lights in their optimal positions for best lighting in the room. Some example layouts are provided for the client or user to see how different positions affect the light in the space. Furthermore, the effect of natural daylight on the spaces can be viewed in the simulation. Figure 2 shows some lighting simulation analysis from different perspectives.

\subsubsection{Acoustic Simulations}

The acoustic module of DIVERCITY offers users the ability to automatically read the CAD-model, to interactively change materials of the building components (walls, floors...) and to "listen" to the acoustic environment inside a building, taking into account sound scenes inside and outside the room.

Acoustic simulation enables the user to have a realistic experience of the acoustic of a space in building. It yields sounds that can be perceived by the user and used very easily to evaluate the project from acoustic point of view.

\subsubsection{Thermal Simulations}

The thermal module of DIVERCITY offers users the ability to automatically read the CAD-model yielded by a CAD tool supporting IFC export, to interactively change materials of the building components (walls, floors,) and to simulate variation of temperatures in different rooms and calculate exploitation costs.

The application enables the user to obtain quick feedback on the thermal performances of the building including a realistic visualisation of the temperatures in each thermal space and relevant information about exploitation costs related to the HVAC system. The client or user can change the materials of the building in order to reach a compromise between comfort conditions on one hand and exploitation costs on the other. Thermal analysis can be a complex task taking into account diverse parameters such as building geometry, climatic environment, HVAC systems, behaviour of the occupants, infiltration and natural ventilation, air quality and pollutant transport (Shelbourn et al, 1999).

\subsection{Construction Workspace Applications}

The DIVERCITY construction workspace aims at providing functionality to allow for rehearsing, evaluation, and optimisation of the construction planning stage. It can be thought of as testing the constructibility of a building by assessing both temporal and spatial aspects resulting from a planned schedule so as to identify and resolve potential conflicts that would otherwise impose high costs if treated at later stages (Fernando et al, 2001).

\subsubsection{Site Planning and Analysis}

This application aims to design a modelling and simulation platform for supporting the 
construction site analysis stage, and allow the evaluation and optimisation of the construction site layouts. In particular, it addresses the space planning aspects by assisting with the representation and management of spatial requirements in the construction site (Tawfik \& Fernando, 2001). The main functions that are carried out by the siteplanning application are as follows.

- Site layout initialisation: initial layout is generated by the user interacting with a VR environment and populating the construction site with different spaces (vehicles, building components, temporary facilities, etc), taking into account schedule information. Alternatively, an initial site layout is constructed from GIS or CAD data.

- Safety analysis: determines the hazard zones of site spaces such as cranes, vehicles and equipment, according to their variable degree of risk and dimensions.

- Space analysis: defines movement path and fields of vision for people and vehicles, and evaluates accessibility and visibility in the site.

- Optimisation: the generation of a favourable spatial arrangement of the site using an optimisation algorithm, a user defined risk minimisation, space use -efficiencymaximisation and travelling cost minimisation criteria.

- The Buildability Schedule provides information on the changing spatial dimensions of objects in the site over time, such as the size of the building or the material store, etc. This information could then be feedback to the site modelling and optimisation modules, to evaluate the site layout at different stages of the construction period on the site.

\subsubsection{Visual Product Chronology}

The second application of the construction workspace in DIVERCITY is a 4D VR simulation application namely Visual Product Chronology that step by step shows how the progress a construction project will look like in practice. The application links a standard IFC based 3D building model with associated construction schedule, which can be prepared with off the shelf project management software package (Fernando et al, 2001). The first basic process of using 4D simulation application is about linking Building Model with Project Task Model. The IFC building product model provides the standard for storing all this information. The additional processes firstly cover the situation where task timings have been changed using project management software package and there is a need to update the IFC 4D building model with this data.
Secondly, the additional processes facilitate the conversions between IFC task model and the used project management software package. Afterwards, updated 4D IFC model is converted to VRML format for simulation. Subsequently, the software makes it possible to simulate the building schedule for example, day by day. The stage that will be reached at the construction site can be seen on the computer display. The easiest way to use the software is to access it by using an Internet browser, but it is also possible to take advantage of it in virtual-reality studios. Figure 3 shows the process flow and simulation display of the Visual Product Chronology.

\subsection{Communication and Integration}

\subsubsection{Communication Layer}

The Communication Layer is at the heart of the distributed features of the DIVERCITY system. It provides support to allow virtual collaborative spaces at geographically distant sites to work together.

The communication layer of DIVERCITY employs XML as the distribution layer for the exchange of information. The implementation of communication layer is based on SOAP (Simple Object Access Protocol) Internet protocol. One of the main advantages of SOAP protocol is that it deals with proxies and firewalls, which are often very strict in the industry domain (Da Dalto \& Gobbetti, 2001). The communication layer provides the followings.

- Communication between heterogeneous systems, architectures and languages.

- Robust and secure messages transfer

- Time performances to allow real time collaboration (only for specific messages-3D scene motions and updates).

- Multi-user management

including identifications and access control.

\subsubsection{Product Modelling}

IFC has been used as the product modelling technology, which was developed by the International Alliance for Interoperability (http://www.iai.org.uk). The IFC defines a single object model of buildings shared by all IFC compatible applications. IFC project models enable the users to exchange information accurately and error-free (Christianson, et al 2002). That is to say, an IFC sketch produced in the Pre CAD application can be distributed over the communication layer and loaded to the other DIVERCITY applications without any 
duplications and repetitions throughout the project lifecycle.

As well as the IFC, ISO Part 42 of STEP (Standard for the Exchange of Product Data) is also employed to keep track of a geometric representation within the DIVERCITY kernel. Basing our common geometric representation on this standard has enforced common comprehension of geometry by different Data Structuring Layers (Christianson et al, 2002).

\section{CONCLUSION}

The paper first explained the vision for integration and collaboration for the construction industry. Afterwards, it described the DIVERCITY R\&D effort. The DIVERCITY project aimed at developing innovative workspace technologies for the briefing and design phases of the lifecycle. The DIVERCITY system incorporates six main applications, each of which responds some special end user requirements from early briefing to the detailed design and the construction monitoring stages. Those applications can run comfortably within the DIVERCITY framework. Output of one application can be distributed and be input to another application, which denotes a seamless integration and collaboration for the stakeholders.

The DIVERCITY project has succeeded in gathering science and industry in a collaborative, exploitative and enriching workspace. The traditional barriers between special disciplines were broken down to establish collaboration scenarios based on mutual visions (Christianson et al, 2002).

\section{REFERENCES}

Alshawi, M. (1996) “SPACE: integrated environment” Internal Paper, University of Salford, July 1996.

Aouad, G., Marir F., Child T., Brandon P. and Kawooya A. (1997) "Construction Integrated Databases- Linking design, planning and estimating, The OSCON approach”; International Conference on the Rehabilitation and Development of Civil Engineering Infrastructures. American University of Beirut, pp 52-60, June 1997

Barrett P., Stanley C., (1999) "Better Construction Briefing”, University of Salford, Blackwell Science.

Aspin R., Fernando T., (2002) "Development and Exploration of Conceptual Building Function-to-Form Relationships”. ECPPM 2002 European Conference of Product and Process Modelling. eWork and eBusiness in AEC, Portoroz, Slovenia.. 9-11 September 2002

Brandon, P.S., (1999) "Product Process Development in 2000 Beyond”, Berkeley-Stanford CE\&M Workshop, Stanford 1999.

Christiansson P., Da Dalto Laurent, Skjaerbaek J. O., Soubra S., Marache M., 2002, " Virtual Environments for the AEC sector - The DIVERCITY experience ". ECPPM 2002 Proceedings European Conference of Product and Process Modelling. eWork and eBusiness in AEC. (Editors: Ziga Turk, Raimar Scherer). Swtes \& Zeitlinger Publishers, Lisse The Netherlands. ISBN 90 5809507 X. 9-11 September 2002, Portoroz, Slovenia. (pp. 49-55)

Christianson P., Da Dalto L., Skjaerbaek, J. O., Soubra S., Marache M., (2002) Virtual Environments for the AEC sector, ECPPM conference, Slovenia, September 2002, paper 465

Christiansson P, Svidt K, Skjærbæk J O, Aaholm R, (2001). "User requirements modelling in design of collaborative virtual reality design systems", International Conference on Construction Information Technology, Mpumalanga, South Africa

Construct IT (2000), Construction Modelling Methodologies for Intelligent Information Integration (COMMIT), Construct IT Centre of Excellence, UK, ISBN 1-900491-76-1

Coudret F., Lombardo J.C., Marache M., Soubra S., (2001) A VR application for the Construction Industry, Virtual Reality International Conference, Laval Virtual, May 16th - 18th 2001

Da Dalto L., Gobbetti E. (2001), DIVERCITY Deliverable 16\&17: System Architecture; Multiresolution Module and Communication Services.

Divercity Handbook (2003), “The Divercity Project: A Virtual Toolkit for Constructin Briefing, Design and Management”, University of Salford, UK.

Faraj I., Alshawi, M., (1999), “A Modularised Integrated Computer Environment For the Construction Industry: SPACE, University of Salford, UK, http://itcon.org/1993/3/

Fernando T., Kähkönen K., Leinonen, J. Murray N., Tawfik H., (2001) Facilitation of collaborative communication for building construction with Virtual Reality Technology, Conference on Applied Virtual Reality in Engineering \& Construction Applications of Virtual Reality, October 4-5, 2001Gothenburg, Sweden, (pp. 117).

IFC http://www.iai.org.uk

Issa, R., (1999) "Virtual Reality: A Solution to Seamless Technology Integration in the AEC Industry”, Berkeley-Stanford CE\&M Workshop, Stanford 1999.

Kartam N.A., (1994). "Interactive system for integrating CAD and computer based construction systems”, In: Microcomputers in civil engineering, Vol 9, pp.41-51.

Kiviniemi, A., Laitinen, J., Lautanala, M., (1999) “Defining a Research Agenda for AEC”, BerkeleyStanford CE\&M Workshop, Stanford 1999.

Sarshar M., Christiansson P, and Winter J (2004) Towards Virtual Prototyping in the Construction Industry: The Case Study of the DIVERCITY project. In: Brandon P, Li H, Shaffii N, Shen Q (Eds), INCITE Conference, 1821 February 2004, Langkawi Malaysia. Designing, Managing and Supporting Construction Projects through Innovation and IT solutions, Vol. 1, 581-8. 
Sarshar, M., Betts, M., Abbott, C., Aouad, G.,(2000) “A Vision for Construction IT 2005-2010”, RICS (Royal Institute of Chartered Surveyors) Research Series, Dec 2000

Sarshar, M., Tanyer, A., Aouad, G., Underwood, J., (2002) “A Vision for Construction IT 2005-2010: Two Case Studies”, Engineering, Construction \& Architectural Management, Issue 2, April, 2002.

Sawhney, A., (1999) "Research and Development Plan for the AEC Industry”, Berkeley-Stanford CE\&M Workshop, Stanford 1999.

Shelbourn M., Soubra S. \& J. Martin, (1999) DIVERCITY Project Deliverable 8, Design Review Workspace
Shi, J.J. (1999) "Computer Simulation in AEC and its Future Development”, Berkeley-Stanford CE\&M Workshop, Stanford 1999.

Tawfik, H., Fernando, T. (2001), "A simulation environment for construction site planning", The $5^{\text {th }}$ IEEE International Conference on Information Visualisation, London, UK, 25- 27 July 2001

Thabet, W., (1999) "Design-Construction Integration Through Virtual Construction for Improved Constructability”, Berkeley-Stanford CE\&M Workshop, Stanford 1999.

Watson, I.D., \& Marir, F. (1994) "Case-Based Reasoning: A Review.” The Knowledge Engineering Review, Vol. 9(iii), pp. 327-54.

\section{PHOTOGRAPHS AND FIGURES}

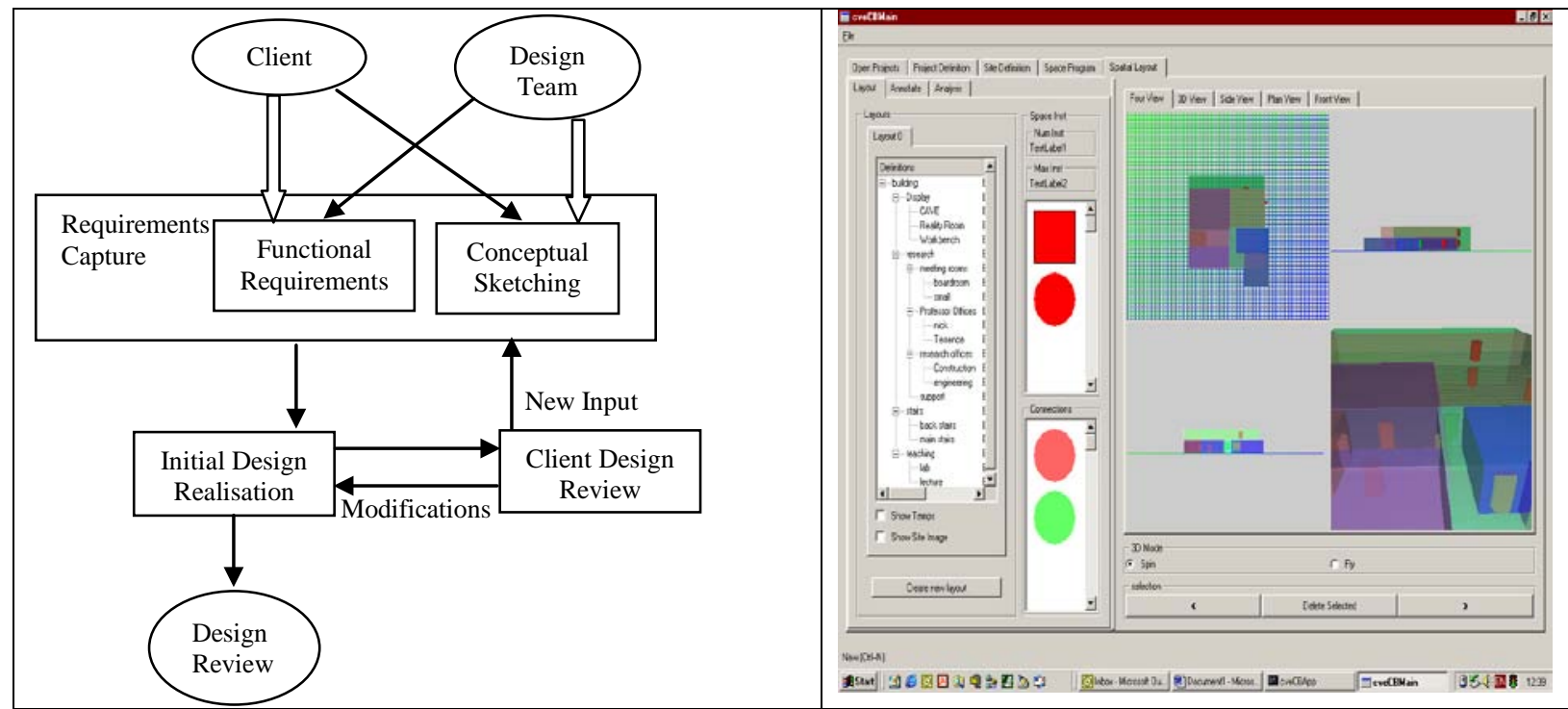

Figure 1: DIVERCITY Client Briefing Process and Pre CAD VR Environment for Client Briefing

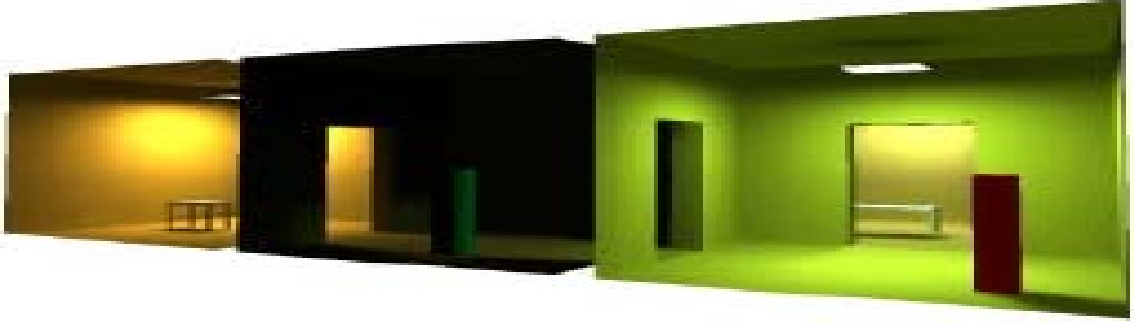




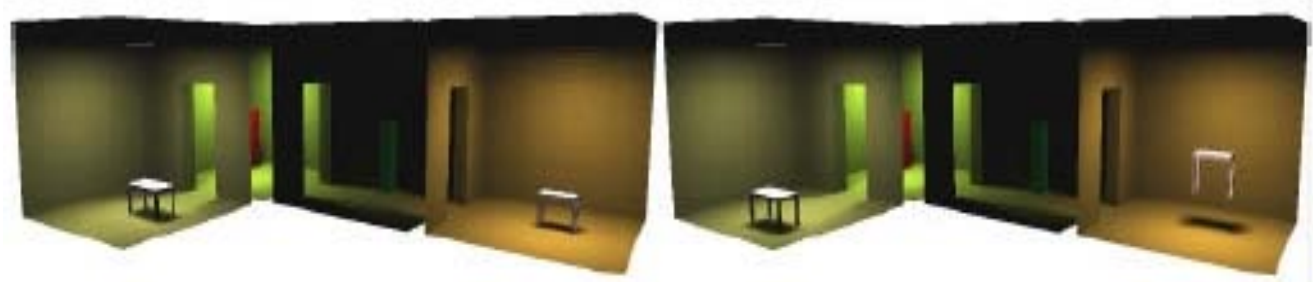

Figure 2: Examples of a rendered scene and object motion in the lighting Simulation

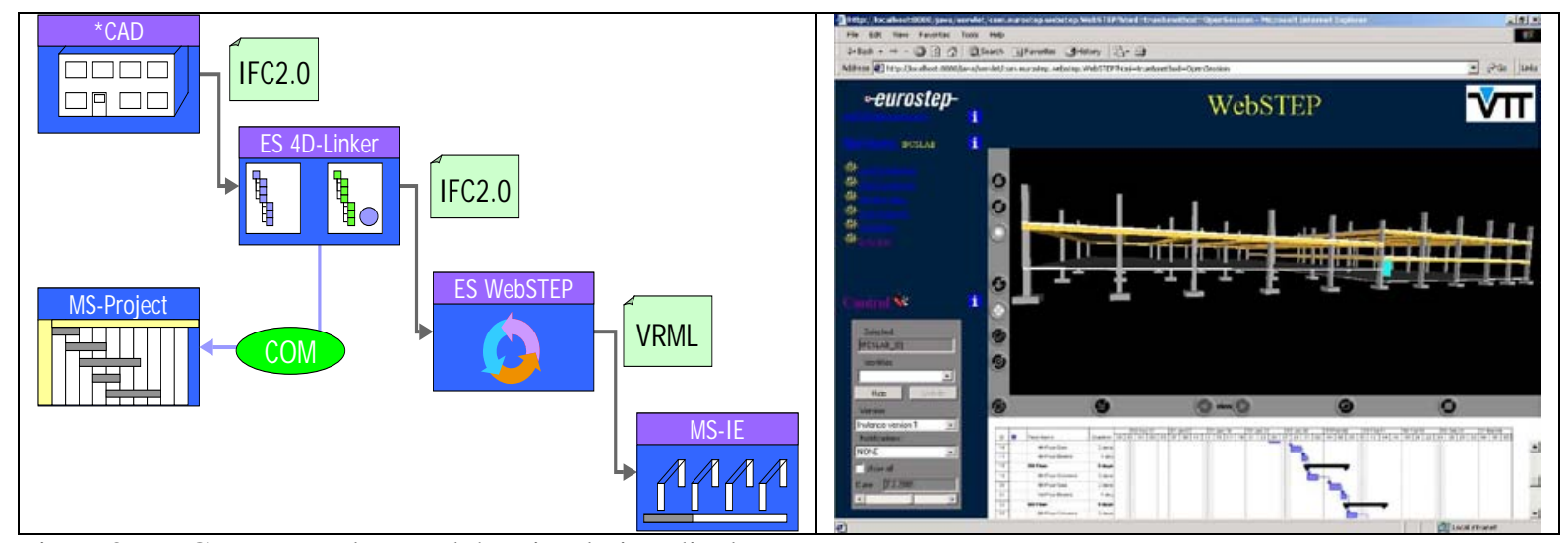

Figure3: VPC Process Flow and 4D simulation display 